\title{
Análisis de la experiencia de consumo, basado en estudios de neuromarketing
}

\author{
Analysis of the experience of consumption, based on neuromarketing studies
}

Diego Marcelo Almeida López ${ }^{1}$, César Antonio Oña Velasteguí ${ }^{2}$, Yolanda Patricia Moncayo Sánchez ${ }^{3}$, Denise Liliana Pazmiño Garzón ${ }^{4} \&$ Marco Vinicio Carrión Torres ${ }^{5}$.

\begin{abstract}
DOI: https://doi.org/10.33262/cienciadigital.v3i3.1.700

The main objective of this research is to analyze the neuromarketing variables applied to customer service in the food and beverage businesses. The problem that arises for the businesses of the catering industry worldwide is to know deeply how are their consumers and what expectations they have when they go to eat at a restaurant; so it is necessary that in this business class new strategies are developed based on the satisfaction of the true needs of the client, generating consumption situations based on emotions. In the development of the investigation a survey was applied to 383 external clients of the company, to know their perceptions regarding the service offered by the business incorporating neuromarketing variables; an experimental meal was also offered to eight people where, through an EEG, it was possible to measure the brain waves of the subjects and observe how the stimuli presented to them affected their emotions. The results obtained through statistical analysis showed the influence of the demography and psychography of consumers on their experience in the business. In the results of the experimental meal it was possible to observe peaks of emotions in the moments where the customers were stimulated by presenting them with one dish or
\end{abstract}

${ }^{1}$ Escuela Superior Politécnica de Chimborazo, Facultad de Administración de Empresas, Riobamba, Ecuador. diego.almeida@espoch.edu.ec

${ }^{2}$ Baltimore Bar Cafetería, Riobamba, Ecuador. el_cesitar18@ @otmail.com

${ }^{3}$ Escuela Superior Politécnica de Chimborazo, Facultad de Administración de Empresas Riobamba, Ecuador. yolanda.moncayo@espoch.edu.ec

${ }^{4}$ Escuela Superior Politécnica de Chimborazo, Facultad de Administración de Empresas Riobamba, Ecuador. denise.pazmino@espoch.edu.ec

${ }^{5}$ Universidad de las Fuerzas Armadas, Riobamba, Ecuador. mvct1980@ hotmail.com 
another and also by trying them; through the analysis of these results, improvement areas are identified from which the service offered by "Baltimore Bar Cafeteria" will be improved.

Keywords: Neuromarketing, restaurant, perceptions, expectations, emotions.

\section{Resumen}

La presente investigación tiene como objetivo principal analizar las variables del neuromarketing aplicadas al servicio al cliente en los negocios de alimentos y bebidas. La problemática que se plantea para los negocios de la industria de la restauración a nivel mundial está en conocer profundamente como son sus consumidores y qué expectativas tienen cuando van a comer a un restaurante; por lo que se hace necesario que en esta clase de negocios se desarrollen nuevas estrategias basadas en la satisfacción de las verdaderas necesidades del cliente generando situaciones de consumo basadas en las emociones. En el desarrollo de la investigación se aplicó una encuesta a 383 clientes externos de la empresa, para conocer sus percepciones respecto el servicio ofertado por el negocio incorporando las variables del neuromarketing; se ofreció además una comida experimental a ocho personas donde mediante un EEG se pudo medir las ondas cerebrales de los sujetos y observar de qué manera los estímulos que se les presentaba afectaban sus emociones. Los resultados que se obtuvieron mediante el análisis estadístico demostraron la influencia que tiene la demografía y psicografía de los consumidores en su experiencia dentro del negocio. En los resultados de la comida experimental se pudo observar picos de emociones en los instantes donde se estimulaba a los clientes presentándoles un plato u otro y también al probarlos; mediante el análisis de estos resultados se identifican áreas de mejora a partir de la cuales se mejorará el servicio ofertado por "Baltimore Bar Cafetería".

Palabras claves: Neuromarketing, restaurante, percepciones, expectativas, emociones.

\section{Introducción.}

Mediante el neuromarketing se puede analizar la psicología tanto a nivel consciente como inconsciente, los patrones y conductas de los consumidores, con el fin de conocer mediante diversas técnicas como reaccionan los mismos a estímulos que ofrecen las empresas y ver cómo perciben los productos de tal manera que se pueda satisfacer sus necesidades de manera exitosa. Atwal (2009) sostiene que: "La generación de experiencias se ha convertido en una de las piedras angulares en algunos avances recientes y en áreas como las de la venta al retail, turismo y eventos.".

El neuromarketing ya se aplica en negocios de este mismo tipo en diferentes partes del mundo; según Braidot (2011) "Burger King incorporó hace unos años en sus locales un sistema que emitía un aroma a carne a la parrilla y otras empresas se han sumado a este tipo de iniciativas.". 
En el Ecuador, "Urko" del Chef Juan Sebastián Pérez, aplicando técnicas de cocina de vanguardia ha logrado presentar una nueva propuesta experiencial a sus clientes.

Para los negocios del sector gastronómico, es fundamental el mejoramiento de la calidad en los productos y los servicios que presta. El Neuromarketing está muy lejos del conocimiento de propietarios y chefs de muchos restaurantes en Ecuador, desconociendo que mediante la creación de experiencias, se puede pasar de vender simples productos a vender emociones y momentos.

En la ciudad de Riobamba la oferta gastronómica es variada y en los últimos años se ha visto un notable crecimiento de los negocios del servicio de alimentos y bebidas. Cafeterías, bares y restaurantes han ido apareciendo en el centro y los alrededores de la ciudad; lamentablemente ninguno maneja técnicas de neuromarketing en sus menús ni en el tratamiento que brindan a sus clientes.

La empresa "Baltimore Bar Cafetería" tampoco cuenta con un plan de estrategias de neuromarketing basadas en el conocimiento profundo de sus clientes externos.

El objetivo de la investigación es conocer si las características demográficas y psicográficas de los clientes afectan sus experiencias de consumo, y cuáles son las reacciones de los clientes ante los estímulos brindados por la comida; para de este modo desarrollar nuevas estrategias para satisfacer las necesidades de los consumidores externos, generando de esta manera situaciones de consumo basadas en las emociones.

A continuación se presentan algunas teorías y enfoques teóricos acerca del neuromarketing:

\section{El Neuromarketing y el análisis de las emociones}

El neuromarketing es una ciencia que busca explicar el proceso de toma de decisiones y el comportamiento de los consumidores del mercadeo tradicional (Braidot, 2013). En otras palabras, el neuromarketing "estudia los procesos mentales que dan explicación a la percepción, conducta y toma de decisiones de los clientes en un contexto de marketing" (Baptista, León y Mora, 2010 p.18).

El neuromarketing implementa técnicas del campo de las neurociencias en la investigación del mercadeo tradicional (Monge y Fernández, 2011), apuntándole a explorar "el 95\% de los pensamientos, emociones y aprendizajes que se producen en la mente inconsciente, sin que nos demos cuenta de ello" (Zaltman, 2004 p.77).

De acuerdo con este autor, en la mente inconsciente se da el proceso de toma de decisiones de consumo, ya que los juicios inconscientes son los que orientan a los conscientes, y concluye que los métodos tradicionales de investigación del mercadeo solo vislumbran el 5\% consciente. 


\section{La importancia de las emociones dentro del comportamiento del consumidor}

Cuando se habla del marketing de experiencias, se refiere a uno de los enfoques del marketing que no solo se preocupa de la venta de un producto determinado, si no que se encarga también de generar diferentes sentimientos en los consumidores.

Hay que tomar en cuenta que a través del tiempo la mercadotecnia ha ido adquiriendo diferentes visiones, antes el concepto de una propuesta se concentraba en las características del producto y se decía que los consumidores preferirían aquellos con mejor calidad o mejor rendimiento; después se pasó al marketing de tipo social en donde hay mayor interés en proteger e incrementar el bienestar del consumidor y de la sociedad. De esta manera se llegó al marketing de experiencias, el enfoque del marketing que se preocupa de que los clientes no adquieran solo productos, sino que también adquieran experiencias; según Pine II y Gilmore (2002) las experiencias antes estaban consideradas como parte integrante del servicio, pero esto no quiere decir que antes no existían.

"Cuando se contrata un servicio, se compra un conjunto de actividades intangibles, mientras que, en una experiencia, el cliente paga por pasar el tiempo disfrutando de esa serie de eventos memorables que la empresa le ofrece a cambio de comprometerlo personalmente" (Pine II y Gilmore, 2002, p.8)

En el ámbito de las empresas de alimentos y bebidas, se debe tomar en cuenta las exigencias que tienen los clientes para que estas se incluyan en las propuestas de cada negocio.

Según Pine II y Gilmore (2002) "las experiencias son memorables, generan sensaciones personales, emocionales, físicas, intelectuales, incluso espirituales. Por lo tanto, nunca habrá dos experiencias iguales, ya que cada presentación de la experiencia entra en interacción con el estado mental del individuo antes de vivirla".

Por esto es necesario conocer y entender las expectativas y percepciones que tienen los clientes con respecto a las experiencias en una empresa.

\section{Marketing tradicional vs. Marketing experiencial}

La incorporación de la óptica experiencial en el marketing con el objeto de avanzar en el conocimiento del comportamiento de compras de los consumidores se produce en los años 80, momento en el que se empieza a tomar conciencia del valor de las emociones como elemento determinante en el proceso de compra. Este enfoque supone una orientación postmoderna del marketing, girando el carácter central del mismo en torno a la consideración de las personas como individuos emocionales interesados en lograr experiencias de consumo placenteras y agradables. Es más, una experiencia agradable y única tendrá un carácter personal dependiendo del sujeto y de la situación en la cual éste la reciba (Walls et al., 2011). 
Actualmente, se afirma que un enfoque tradicional del marketing, centrado en las funcionalidades y calidades del producto, es insuficiente para ofrecer al consumidor experiencias de consumo inolvidables y estimulantes (Hosany y Witham, 2010; Tsiotsou y Ratten, 2010).

Por ello, el Marketing Experiencial incide al consumidor, centrándose en generar una experiencia agradable no solo en el momento de la compra si no en diversas situaciones, incluyendo el consumo y el post consumo, recurriendo para ello a la creación de emociones, sentimientos y pensamientos consecuencias de la interacción entre la marca o empresa y el cliente.

El núcleo central del Marketing Experiencial es la "experiencia del consumidor", de ahí que, como paso previo a su conceptualización, haya que abordar el origen conceptual del término “experiencia”. Según el Diccionario de la Real Academia Española de la Lengua (R.A.E), una experiencia puede definirse como "la circunstancia o acontecimiento vivido por una persona".

Los factores que han permitido la evolución del Marketing Tradicional al Marketing Experiencial son (Schmitt 1999 y 2006):

- La omnipresencia de la tecnología de la información, determinante para propiciar el contacto y una conexión entre la empresa y el cliente, como medio para la creación y puesta en valor de las experiencias a entregar al cliente.

- La supremacía de la marca. Gracias al impulso de las tecnologías de la información se podrá disponer de información de un modo inmediato, siendo las marcas un medio para facilitar experiencias al cliente.

- Predominio de las comunicaciones y el esparcimiento. Se impondrán unas comunicaciones fluidas entre la empresa y el cliente fruto de una mayor orientación de las organizaciones hacia el cliente final.

En la siguiente tabla se puede observar las diferencias más grandes entre el marketing tradicional y el marketing de experiencias:

Tabla 1: Diferencias entre el marketing tradicional y el marketing de experiencias

\section{MARKETING TRADICIONAL}

- Se basa en las características del producto y los beneficios que éste crea.

- Los clientes se basan en la razón a la hora de la compra.

- Se centra más en perseguir al cliente para que compre.

- Busca tener contactos esporádicos con el cliente

Elaborado por: Autores

\section{MARKETING DE EXPERIENCIAS}

- Se basa en experiencias del cliente creando un vínculo entre el cliente, el producto y la marca.

- Los clientes toman la decisión de compra a través de emociones.

- Se centre en que el momento de adquisición y uso del producto sea más eficaz para que la marca influya.

- Busca una relación con el cliente ininterrumpida. 


\section{Metodología}

En la investigación se analizó el desarrollo de las variables plateadas a través del tiempo, convirtiéndola en una investigación de tipo experimental.

El tipo de diseño fue longitudinal, el enfoque de la presente investigación es de carácter cualitativo puesto que tiene como objetivo la descripción de las cualidades del fenómeno a investigar; como indica Dzul (2014) este enfoque "Trata de conocer los hechos, procesos, estructuras y personas en su totalidad"; de tal manera que así se analizará la percepción de los clientes de "Baltimore Bar Cafetería".

La población fue objeto de este estudio, estuvo compuesta por hombres y mujeres de la población económicamente activa (PEA) de la ciudad de Riobamba, dato que según el INEC la conforman 123.457 personas.

La aplicación de la fórmula determinó un tamaño de muestra de 383 personas. El instrumento que se utilizaron para la recolección de datos fuer el cuestionario en el cual se recabó datos demográficos y psicográficos de los clientes, así como la experiencia de los clientes dentro del negocio.

\section{Resultados}

\section{A. Factores demográficos y psicográficos de los clientes de Baltimore}

Tabla 3: Aspecto demográfico - Género

\begin{tabular}{lrrrr}
\hline & Frecuencia & Porcentaje & $\begin{array}{c}\text { Porcentaje } \\
\text { válido }\end{array}$ & $\begin{array}{r}\text { Porcentaje } \\
\text { acumulado }\end{array}$ \\
\hline MASCULINO & 144 & 37,6 & 37,6 & 37,6 \\
\hline FEMENINO & 239 & 62,4 & 62,4 & 100,0 \\
\hline Total & 383 & 100,0 & 100,0 & \\
\hline
\end{tabular}

Fuente: Análisis del Software SPSS versión 23

Elaborado por: Los autores

Tabla 4: Aspecto Demográfico-Edad

\begin{tabular}{lrrrr}
\hline & Frecuencia & Porcentaje & $\begin{array}{l}\text { Porcentaje } \\
\text { valido }\end{array}$ & $\begin{array}{l}\text { Porcentaje } \\
\text { acumulado }\end{array}$ \\
\hline ENTRE 20 Y 25 AÑOS & 121 & 31,6 & 31,6 & 31,6 \\
\hline ENTRE 26 Y 30 AÑOS & 138 & 36,0 & 36,0 & 67,6 \\
\hline ENTRE 31 Y 35 AÑOS & 67 & 17,5 & 17,5 & 85,1 \\
\hline ENTRE 36 Y 40 AÑOS & 18 & 4,7 & 4,7 & 89,8 \\
\hline ENTRE 41 Y 45 AÑOS & 39 & 10,2 & 10,2 & 100,0 \\
\hline Total & 383 & 100,0 & 100,0 & \\
\hline
\end{tabular}

Fuente: Análisis del Software SPSS versión 23

Elaborado por: Los autores 
Tabla 5: Aspecto Demográfico-Nivel de Estudios

\begin{tabular}{lrrrr}
\hline & Frecuencia & Porcentaje & $\begin{array}{c}\text { Porcentaje } \\
\text { válido }\end{array}$ & $\begin{array}{l}\text { Porcentaje } \\
\text { acumulado }\end{array}$ \\
\hline BACHILLER & 15 & 3,9 & 3,9 & 3,9 \\
\hline TERCER NIVEL & 265 & 69,2 & 69,2 & 73,1 \\
\hline CUARTO NIVEL & 103 & 26,9 & 26,9 & 100,0 \\
\hline Total & 383 & 100,0 & 100,0 & \\
\hline
\end{tabular}

Fuente: Análisis del Software SPSS versión 23

Elaborado por: Los autores

\section{B. Factores de la experiencia del cliente}

El objetivo de esta parte es conocer, como perciben los clientes, ciertos aspectos de "Baltimore Bar Cafetería", se realizaron preguntas acerca del ambiente del negocio.

Tabla 6: Percepción del diseño interior de Baltimore

\begin{tabular}{lrrrr}
\hline & Frecuencia & Porcentaje & $\begin{array}{c}\text { Porcentaje } \\
\text { válido }\end{array}$ & $\begin{array}{c}\text { Porcentaje } \\
\text { acumulado }\end{array}$ \\
\hline MUY EN DESACUERDO & 10 & 2,6 & 2,6 & 2,6 \\
\hline EN DESACUERDO & 43 & 11,2 & 11,2 & 13,8 \\
\hline NI DEACUERDO NI EN & 126 & 32,9 & 32,9 & 46,7 \\
DESACUERDO & 149 & 38,9 & 38,9 & 85,6 \\
\hline DE ACUERDO & 55 & 14,4 & 14,4 & 100,0 \\
\hline MUY DE ACUERDO & 383 & 100,0 & 100,0 &
\end{tabular}

Fuente: Análisis del Software SPSS versión 23

Elaborado por: Los autores

Tabla 7: Percepción del diseño exterior de Baltimore

\begin{tabular}{lrrrr}
\hline & & & $\begin{array}{c}\text { Porcentaje } \\
\text { válido }\end{array}$ & $\begin{array}{l}\text { Porcentaje } \\
\text { acumulado }\end{array}$ \\
\hline MUY EN & 27 & 7,0 & 7,0 & 7,0 \\
DESACUERDO & 74 & 19,3 & 19,3 & 26,4 \\
\hline EN DESACUERDO & 120 & 31,3 & 31,3 & 57,7 \\
\hline NI DEACUERDO NI & 117 & 30,5 & 30,5 & 88,3 \\
EN DESACUERDO & 45 & 11,7 & 11,7 & 100,0 \\
\hline DE ACUERDO & 383 & 100,0 & 100,0 & \\
\hline MUY DE ACUERDO & & & & \\
\hline Total & & & & \\
\hline
\end{tabular}

Fuente: Análisis del Software SPSS versión 23

Elaborado por: Los autores 
Tabla 8: Percepción del aroma del restaurante

\begin{tabular}{|c|c|c|c|c|}
\hline & Frecuencia & Porcentaje & $\begin{array}{l}\text { Porcentaje } \\
\text { válido }\end{array}$ & $\begin{array}{l}\text { Porcentaje } \\
\text { acumulado }\end{array}$ \\
\hline MUY EN DESACUERDO & 14 & 3,7 & 3,7 & 3,7 \\
\hline EN DESACUERDO & 52 & 13,6 & 13,6 & 17,2 \\
\hline $\begin{array}{l}\text { NI DEACUERDO NI EN } \\
\text { DESACUERDO }\end{array}$ & 127 & 33,2 & 33,2 & 50,4 \\
\hline DE ACUERDO & 152 & 39,7 & 39,7 & 90,1 \\
\hline MUY DE ACUERDO & 38 & 9,9 & 9,9 & 100,0 \\
\hline Total & 383 & 100,0 & 100,0 & \\
\hline
\end{tabular}

Fuente: Análisis del Software SPSS versión 23

Elaborado por: Los autores

Tabla 9: Percepción sobre la presentación de los platos del menú

\begin{tabular}{lrrrr}
\hline & Frecuencia & Porcentaje & $\begin{array}{c}\text { Porcentaje } \\
\text { válido }\end{array}$ & $\begin{array}{c}\text { Porcentaje } \\
\text { acumulado }\end{array}$ \\
\hline MUY EN DESACUERDO & 21 & 5,5 & 5,5 & 5,5 \\
\hline EN DESACUERDO & 66 & 17,2 & 17,2 & 22,7 \\
\hline NI DEACUERDO NI EN & 134 & 35,0 & 35,0 & 57,7 \\
DESACUERDO & 118 & 30,8 & 30,8 & 88,5 \\
\hline DE ACUERDO & 44 & 11,5 & 11,5 & 100,0 \\
\hline MUY DE ACUERDO & 383 & 100,0 & 100,0 & \\
\hline Total & & & & \\
\hline
\end{tabular}

Fuente: Análisis del Software SPSS versión 23

Elaborado por: Los autores

Tabla 10: Percepción de la música

\begin{tabular}{lcccc}
\hline & Frecuencia & Porcentaje & $\begin{array}{c}\text { Porcentaje } \\
\text { válido }\end{array}$ & $\begin{array}{c}\text { Porcentaje } \\
\text { acumulado }\end{array}$ \\
\hline MUY EN DESACUERDO & 15 & 3,9 & 3,9 & 3,9 \\
\hline EN DESACUERDO & 49 & 12,8 & 12,8 & 16,7 \\
\hline $\begin{array}{l}\text { NI DEACUERDO NI EN } \\
\text { DESACUERDO }\end{array}$ & 176 & 46,0 & 46,0 & 62,7 \\
\hline DE ACUERDO & 97 & 25,3 & 25,3 & 88,0 \\
\hline MUY DE ACUERDO & 46 & 12,0 & 12,0 & 100,0 \\
\hline Total & 383 & 100,0 & 100,0 & \\
\hline
\end{tabular}

Fuente: Análisis del Software SPSS versión 23

Elaborado por: Los autores 
Tabla 11: Percepción de la experiencia

\begin{tabular}{lrrrr}
\hline & & & Porcentaje & \multicolumn{2}{c}{$\begin{array}{c}\text { Porcentaje } \\
\text { acumulado }\end{array}$} \\
\hline MUY EN DESACUERDO & 19 & 5,0 & 5,0 & 5,0 \\
\hline EN DESACUERDO & 27 & 7,0 & 7,0 & 12,0 \\
\hline NI DEACUERDO NI EN & 124 & 32,4 & 32,4 & 44,4 \\
DESACUERDO & 149 & 38,9 & 38,9 & 83,3 \\
\hline DE ACUERDO & 64 & 16,7 & 16,7 & 100,0 \\
\hline MUY DE ACUERDO & 383 & 100,0 & 100,0 & \\
\hline Total & & & & \\
\hline
\end{tabular}

Fuente: Análisis del Software SPSS versión 23

Elaborado por: Los autores

\section{Análisis de la etapa de experimentación}

Para realizar el estudio acerca de cómo reacciona el cerebro de los consumidores del bar ante los estímulos presentados por el menú, se realizó un experimento dirigido a un grupo focal (8 personas) seleccionados al azar. El cual consistía en presentar un menú de comida y medir los estímulos que cada cliente reflejaba frente a éste.

Para el experimento de degustación del menú se les sirvió a los clientes un menú de 3 cursos, el cual constaba de lo siguiente:

Tabla 12: Menú para experimentación

\begin{tabular}{|c|c|}
\hline CURSO DEL MENU & DETALLE \\
\hline ENTRADA & $\begin{array}{c}1 \text { porción de alitas Picantes } \\
1 \text { porción de alitas Baltimore } \\
\text { El plato estuvo acompañado de una porción de } \\
\text { aderezo ranchero. }\end{array}$ \\
\hline FUERTE & $\begin{array}{c}1 \text { porción de Lomo a la Piedra } \\
\text { El Lomo estuvo acompañado de una porción de } \\
\text { vegetales salteados. }\end{array}$ \\
\hline BEBIDA & $\begin{array}{c}\text { Frestea (el té helado de la casa, acompañado con } \\
\text { fresas y hierbas aromáticas); Jugo de mandarina y } \\
\text { hierba buena. }\end{array}$ \\
\hline
\end{tabular}

Elaborado por: Los autores

El análisis de ondas cerebrales se realizó mediante la utilización de "Emotiv Insight" un EEG inalámbrico móvil de 5 canales, el cual graba las ondas cerebrales y las traduce en información significativa que se puede entender. Está diseñado para uso extendido e investigaciones de campo. 
Este equipo es el único en su categoría que ofrece 5 sensores de EEG más 2 sensores de referencia. Esta resolución provee información profunda en la actividad cerebral.

Figura 1: EEG inalámbrico Emotiv Insight

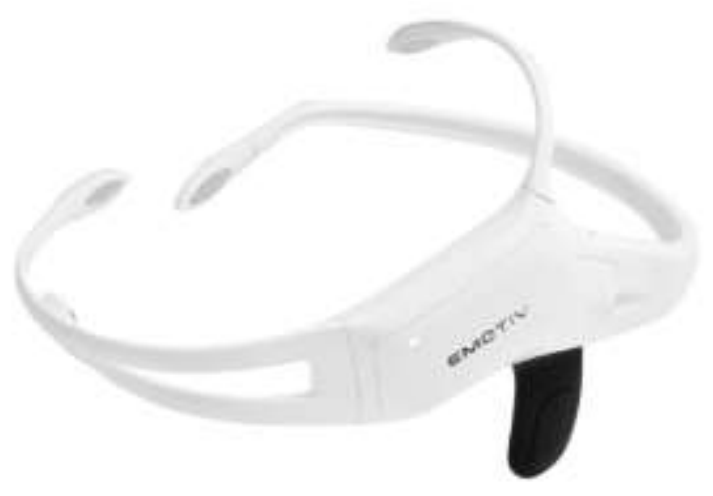

Fuente: https://www.emotiv.com/insight/

Utilizando la aplicación "My Emotiv", se conectó el EEG a un teléfono móvil donde se presentaba el movimiento de las ondas cerebrales, esta aplicación presenta los resultados en base a las siguientes emociones:

- Stress (Estrés): mide que tan cómodo se siente la persona con la actividad que está realizando, mantener los niveles de estrés bajos pueden ayudar a mejorar la concentración y la salud física.

- Engagement (Compromiso): mide que tan inmerso esta la persona en la actividad que está realizando.

- Interest (Interés): mide que tanto a una persona le gusta, o le disgusta lo que está haciendo, los resultados medios son neutrales.

- Excitement (Emoción): Captura el nivel de emoción.

- Focus (Concentración): es la habilidad que tiene la persona de concentrarse en una tarea e ignorar las distracciones.

- Relaxation (Relajación): es la habilidad que tiene una persona de desconectarse y buscar un estado mental calmado.

Los principales puntos de análisis en esta experimentación son el nivel de compromiso, el interés y el nivel de emoción que se generan en el cliente con el estímulo de la comida; en el estudio del neuromarketing es importante conocer los picos de cada una de las emociones presentadas por los clientes y en qué momentos se dieron, como se detallan: 
Tabla 14: Picos de emociones por sujeto

\begin{tabular}{crrrrrr}
\hline \multirow{2}{*}{$\begin{array}{c}\text { No. de } \\
\text { sujeto }\end{array}$} & \multicolumn{6}{c}{ EMOCIONES } \\
\cline { 2 - 7 } & \multicolumn{1}{c}{ Engagement } & \multicolumn{1}{c}{ Interest } & \multicolumn{3}{c}{ Excitement } \\
\cline { 2 - 7 } $\mathbf{+}$ & \multicolumn{1}{c}{$\boldsymbol{+}$} & \multicolumn{1}{c}{$\boldsymbol{~}$} & \multicolumn{1}{c}{$\boldsymbol{~}$} \\
\hline $\mathbf{1}$ & 90 & 7 & 100 & 40 & 75 & 8 \\
\hline $\mathbf{2}$ & 90 & 5 & 98 & 38 & 82 & 15 \\
\hline $\mathbf{3}$ & 92 & 7 & 98 & 39 & 80 & 10 \\
\hline $\mathbf{4}$ & 95 & 5 & 100 & 42 & 80 & 15 \\
\hline $\mathbf{5}$ & 90 & 10 & 100 & 39 & 80 & 10 \\
\hline $\mathbf{6}$ & 90 & 7 & 95 & 38 & 80 & 10 \\
\hline $\mathbf{7}$ & 90 & 7 & 100 & 42 & 80 & 10 \\
\hline $\mathbf{8}$ & 90 & 7 & 100 & 38 & 95 & 10 \\
\hline
\end{tabular}

\section{Elaborado por: Los autores}

Se puede observar que, en el caso del compromiso (engagement) los picos más altos están entre los 90 y los 95 puntos, como se observó muchos de estos picos se produjeron al momento de servir el segundo plato; el pico más bajo de emoción es de 5 puntos. Hablando del interés (Interest) existen picos de hasta 100 puntos en el análisis individual y los picos más bajos se encuentran entre el 38 y 42; cabe indicar que este sentido es el que presenta picos más altos; indicando que la comida genera gran expectativa en el cliente.

Por último, el nivel de emoción (excitement) generado en los sujetos de prueba, tiene picos altos de hasta 95 puntos y el pico más bajo es de 8 puntos sobre 100 .

El grado de interés es el más alto de todos, demostrando que en este sentido la estimulación provocada en los clientes es un poco más significativa. Hay que tomar en cuenta que se notó claros picos con puntos altos de entre 80 y 100 puntos, cuando se decidió servir el segundo curso del menú (lomo a la piedra), de una manera diferente, este nuevo modo de servicio del plato marco un significativo crecimiento en los niveles de interés y compromiso en la prueba, los cuales subían generalmente al comenzar la segunda parte de la prueba.

Se debe trabajar en la manera de servir este platillo aplicando el cambio realizado en la experimentación; y este modo de servicio podría convertirse en único, dándole identidad de marca al plato, agregándole un toque que hará que la experiencia del cliente al probar este platillo sea más placentera.

\section{Comprobación de la hipótesis}

El análisis de los resultados obtenidos en las encuestas y durante la etapa de experimentación con el menú, demuestran que existen diversos estímulos que la empresa puede brindarle al cliente, para mejorar su experiencia dentro del negocio; algunos son conscientes y otros inconscientes. 
La hipótesis general planteada al inicio de la investigación sostiene: "Las variables del Neuromarketing, influyen en la experiencia del consumidor de Baltimore Bar Cafetería"; para analizar la correlación entre las variables del neuromarketing (diseño interior, diseño exterior, aroma del restaurante, música) y la experiencia de los consumidores, se utilizó el coeficiente de correlación de Spearman (rho), el cual mide el nivel de concordancia multivariable.

En la tabla 16, se demuestra que si existe una prueba estadística significativa para asegurar que estos factores influyen de una u otra forma en la percepción del cliente acerca de la experiencia de visitar Baltimore; de tal manera que la hipótesis general ha sido comprobada.

Para analizar la relación entre las variables del neuromarketing y la experiencia de consumo, se ha utilizado el coeficiente de Spearman, que permite medir el grado de correlación entre variables.

Tabla 16: Correlación entre variables neuromarketing- experiencia

\begin{tabular}{|c|c|c|c|c|c|c|c|}
\hline & & 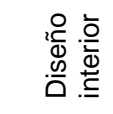 & 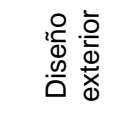 & 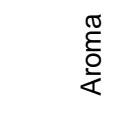 & 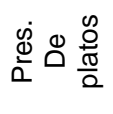 & 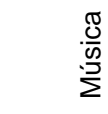 & $\begin{array}{l}\dot{\dot{\Phi}} \\
\dot{\varpi} \\
\dot{x}\end{array}$ \\
\hline \multirow{3}{*}{$\begin{array}{l}\text { Diseño } \\
\text { interior }\end{array}$} & Coef. corre & 1,000 &, $478^{* *}$ &, $397^{* *}$ & $449^{* *}$ & $477^{* *}$ & ,490** \\
\hline & Sig. bilateral & &, 000 &, 000 &, 000 &, 000 & 000 \\
\hline & $\mathrm{N}$ & 383 & 383 & 383 & 383 & 383 & 383 \\
\hline \multirow{3}{*}{$\begin{array}{l}\text { Diseño } \\
\text { exterior }\end{array}$} & Coef. corre &, $478^{* *}$ & 1,000 & $423^{* *}$ &, $541^{* *}$ &, $525^{* *}$ &, $528^{* *}$ \\
\hline & Sig. bilateral &, 000 & &, 000 & , דיד &, 000 &, 000 \\
\hline & $\mathrm{N}$ & 383 & 383 & 383 & 383 & 383 & 383 \\
\hline \multirow[t]{3}{*}{ Aroma } & Coef. Corre &, $397^{* *}$ &, $423^{* *}$ & 1,000 &, $486^{* *}$ &, $348^{* *}$ &, $478^{* *}$ \\
\hline & Sig. bilateral & ,000 &, 000 & &, 000 &, 000 & 000 \\
\hline & $\mathrm{N}$ & 383 & 383 & 383 & 383 & 383 & 383 \\
\hline \multirow{3}{*}{$\begin{array}{c}\text { Pres. De } \\
\text { platos }\end{array}$} & Coef. corre &, $449^{* *}$ &, $541^{* *}$ & $486^{* *}$ & 1,000 & $435^{* *}$ &, $492^{* *}$ \\
\hline & Sig. bilateral & ,000 & , ודo &, 000 & &, 000 & 000 \\
\hline & $\mathrm{N}$ & 383 & 383 & 383 & 383 & 383 & 383 \\
\hline \multirow[t]{3}{*}{ Música } & $\begin{array}{l}\text { Coef. } \\
\text { corre }\end{array}$ &, $477^{* *}$ &, $525^{* *}$ &, $348^{* *}$ &, $435^{* *}$ & 1,000 &, $584^{* *}$ \\
\hline & Sig. bilateral &, 000 & ,000 &, 000 &, 000 & . &, 000 \\
\hline & $\mathrm{N}$ & 383 & 383 & 383 & 383 & 383 & 383 \\
\hline \multirow[t]{3}{*}{ Exper. } & $\begin{array}{l}\text { Coef. } \\
\text { Corre }\end{array}$ &, $490^{* *}$ &, $528^{* *}$ &, $478^{* *}$ &, $492^{* *}$ &, $584^{* *}$ & 1,000 \\
\hline & Sig. bilateral & ,000 & ,000 & ,000 &, 000 &, 000 & \\
\hline & $\mathrm{N}$ & 383 & 383 & 383 & 383 & 383 & 383 \\
\hline
\end{tabular}

Fuente: Análisis del Software SPSS versión 23

Elaborado por: Los autores

Al realizar la correlación se obtiene un resultado de ,000 en la relación variables del marketing-experiencia, y, se concluye que si existe una prueba estadística significativa para asegurar que el neuromarketing influye de una u otra forma en la experiencia, ya que estos valores son inferiores a 0,05 (porcentaje de error) se aceptaría esta premisa. 


\section{Conclusiones}

- El mercado objetivo de "Baltimore Bar Cafetería", está compuesto mayoritariamente por clientes de sexo femenino, en edades comprendidas entre 26 y 30 años con un nivel mínimo de estudios de tercer nivel.

- Lo que se considera como variables del neuromarketing: diseño interior y exterior, aroma del restaurante y estilo de la música; tienen influencia sobre la experiencia del consumidor dentro de la empresa, esto se demostró luego de haber realizado el análisis estadístico requerido; en este sentido el mejor aprovechamiento de estas variables incidirá positivamente en la percepción de los consumidores acerca de su experiencia en el local.

- El uso del EEG, permitió conocer de manera directa, que emociones se presentaban en los sujetos de experimentación al momento de presentarles los estímulos con la comida; los factores con puntos más altos fueron: compromiso, interés, relajación y emoción, dentro de estos ámbitos se presentaron picos de entre 80 a 100 puntos en determinados momentos del servicio de la comida y bebida.

- La aplicación del neuromarketing en la investigación constituye un apoyo efectivo en la gestión comercial al posibilitar el conocimiento de las reacciones de los sujetos a diversas situaciones y estímulos, a partir de lo cual es posible diseñar estrategias efectivas y más precisas para mantener y captar clientes.

\section{Referencias Bibliográficas}

Ariely, D. (2008). Las trampas del deseo: como controlar los impulsos irracionales que nos llevan al error. Boston, Ariel.

Atwal, G., Williams, A. (2009). Luxury brand marketing - The experience is everything! Brand Management, 16(5-6), 338-346.

Braidot, N. (2009). Neuromarketing ¿Por qué tus clientes se acuestan con otros si dicen que les gustas tú? Barcelona, Grupo Planeta (GBS).

Cabrera, S. (2013). Marketing gastronómico. La experiencia de convertir el momento del consumo en un recuerdo memorable. Centro de estudios en diseño y comunicación, 45, 165174.

De Andreis, A. (2012). Neuromarketing: una mirada a la mente del consumidor. Ag-Gnosis, $1(1), 51-57$.

Gomes Teixeira, A., Azevedo Barbosa, M., Gomes De Souza, A. (2013). El sistema de oferta de restaurantes de alta gastronomía una perspectiva sensorial de las experiencias de consumo. Recuperado de: http://www.scielo.org.ar/scielo.php?script=sci_arttext\&pid=S185117322013000200009

Hilke, P., Ramsøy, T., Misavljevic, M. (2012). Branding the brain: A critical review and Outlook. Journal of Consumer Psychology, 22 (2012), 18-36.

Klaric, J. (2012). Estamos ciegos. Lima, Editorial Planeta.

Klaric, J. (2014). Véndele a la mente no a la gente. Lima, Business \& Innovation Institute of America-BIIA, Division Editorial.

Kotler, P. (2000). Marketing Management: Analysis, planning and control. New Jersey, Prentce-Hall. 
Mendieta, J. (2013). Pensamiento racional vs. Pensamiento irracional. Recuperado de: https://www.gabinetedepsicologia-mm.com/2013/04/03/pensamiento-racional-vspensamiento-irracional-en-tiempos-de-crisis/

Miranda, C. (2006). Marketing gastronómico Concepto. Recuperado de: http://mikuisine.blogspot.com/2006/07/marketing-gastronmico-concepto.html

Monreal, A. (2017). Como afecta la iluminación a la manera en que comes. Recuperado de: http://www.lavanguardia.com/vivo/psicologia/20170130/413690279446/como-comemossegun-la-luz.html

Plassmann, H., O’doherty, J., Shiv, B., Y Rangel, A. (2007). Marketing actions can modulate neural representations of experienced pleasantness. Pnas, 105(3), 1050-1054.

Schmitt, B. (2007). Experiential Marketing. Barcelona, Deusto S.A. Ediciones.

Romero, J. (2015). 8 principios de neuromarketing para restaurantes que invitan a gastar más. Recuperado de: http://neuromarketing.la/2015/10/comes-lo-que-quieres-o-lo-que-alguienmas-quiere/ 


\section{PARA CITAR EL ARTÍCULO INDEXADO.}

Almeida López, D. M., Oña Velasteguí, C., Moncayo Sánchez, Y., Pazmiño Garzón, D., \& Carrión Torres, M. (2019). Análisis de la experiencia de consumo, basado en estudios de neuromarketing. Ciencia Digital, 3(3.1), 270-284. https://doi.org/10.33262/cienciadigital.v3i3.1.700

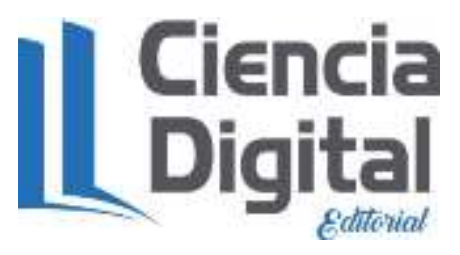

El artículo que se publica es de exclusiva responsabilidad de los autores y no necesariamente reflejan el pensamiento de la Revista Ciencia Digital.

El artículo queda en propiedad de la revista y, por tanto, su publicación parcial y/o total en otro medio tiene que ser autorizado por el director de la Revista Ciencia Digital.
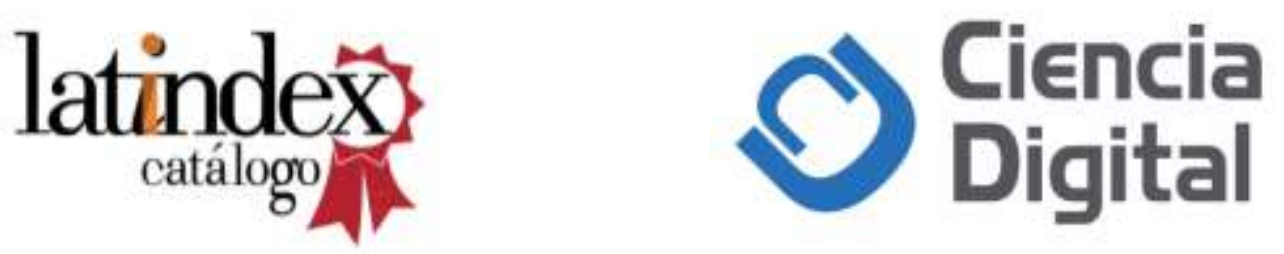